\title{
Serum hepatocyte growth factor as an index of extensive catabolism of patients awaiting liver transplantation
}

\author{
C Miki, A D Mayer, J A C Buckels, K Iriyama, H Suzuki, P McMaster
}

\begin{abstract}
Background-Whole body catabolism as the result of intrahepatic metabolic derangement is common in liver transplant candidates. However, individual nutritional assessment parameters lack sensitivity and specificity in determining energy status of these patients. Recently, serum hepatocyte growth factor (HGF) has been shown to reflect the recovery of hepatic energy metabolism after liver transplantation.
\end{abstract}

Aims-The relation between preoperative levels of serum HGF and metabolic variables was investigated to clarify the clinical value of measuring HGF in evaluations of the catabolism.

Patients/Methods-Blood samples were obtained from 30 liver transplant recipients, and biopsy specimens were taken from each recipient's rectus muscle and the explanted liver. Preoperative serum concentration of HGF was determined. Whole body energy metabolism was assessed by measuring glycogen contents of biopsy specimens and plasma or serum levels of glucose, insulin, total ketone bodies, total carnitine, and amino acids.

Results-Serum HGF concentration was elevated in 22 of 30 patients and correlated with the Child-Pugh score. It showed a negative association with muscle glycogen content, and a positive correlation with serum levels of glucose, total carnitine, and total ketone bodies. Patients with elevated serum HGF concentrations had higher preoperative plasma levels of aromatic amino acids and branched chain amino acids, associated with lower branched chain to aromatic amino acid ratios.

Conclusions-The elevated serum concentration of HGF in liver transplant candidates reflected inhibition of peripheral glucose storage, enhanced lipid oxidation, and increased peripheral release of branched chain amino acids, and thus extensive energy catabolism.

(Gut 1999;44:862-866)

Keywords: hepatocyte growth factor; catabolism; liver; transplantation

Correspondence to: Dr C Miki, Department of Surgery II, Mie University Medical School, Edobashi 2-174, Tsu 514-8507, Japan.

Accepted for publication 13 January 1999

Hypermetabolism associated with proteinenergy malnutrition is present in a significant proportion of liver transplant candidates ${ }^{12}$ and contributes to systemic manifestations of nega- tive energy balance and thus catabolism. As the early postoperative period after liver transplantation is characterised by a state of hypermetabolism associated with hypercatabolism, ${ }^{3}$ and as these aspects of metabolic deterioration have been recognised as risk factors for increased postoperative mortality and morbidity, ${ }^{1}$ preoperative assessment of energy catabolism would be useful for the care of liver transplant recipients.

However, malnutrition has not been quantified previously in the adult transplant population; the definition or assessment of metabolic status in patients with end stage liver disease has been an obstacle. ${ }^{4}$ Measurement of visceral proteins, including albumin, transferrin, retinol binding protein, and prealbumin, provides limited information for nutritional assessments, particularly those of patients with liver disease, in whom the concentrations may reflect reduced hepatic synthesis or the degree of liver damage rather than nutritional status. ${ }^{5}$ Owing to excess fluid accumulation, some anthropometric measures are less sensitive for defining malnutrition.

Hepatocyte growth factor (HGF) is the most potent stimulator of hepatocyte growth and DNA synthesis. It is produced by nonparenchymal hepatic cells ${ }^{67}$ and acts as a growth factor, interacting with specific membrane receptors on the surface of hepatocytes to induce signal transduction. ${ }^{8}$ It plays a key role in the regulation of liver regeneration after partial hepatectomy or hepatocyte damage..$^{9}$ Recently, serum HGF has been reported to be a possible index of catabolism in cancer patients. ${ }^{11}$ Moreover, another study showed that the clearance of serum HGF after transplantation reflects the recovery of hepatic energy metabolism as well as graft viability. ${ }^{12}$ As intrahepatic metabolic changes reflect the whole body catabolic state and can be seen as being adaptative for energy homoeostasis, ${ }^{13}$ preoperative serum HGF levels may reflect the whole body energy status of patients awaiting liver transplantation.

This study was designed to evaluate the relation between preoperative serum HGF levels and the metabolic status of liver transplant candidates and to clarify the clinical value of measuring HGF for evaluating the deterioration of whole body energy metabolism.
Abbreviations used in this paper: HGF, hepatocyte growth factor; AAA, aromatic amino acid; BCAA, branched chain amino acid. 


\section{Patients and methods}

A total of 30 recipients who had undergone orthotopic liver transplantation at The Queen Elizabeth Hospital were enrolled in the study. Twenty normal healthy age and sex matched volunteers were used as controls. The transplants were performed in patients with chronic liver disease (primary biliary cirrhosis in 15, hepatitis $\mathrm{C}$ virus cirrhosis in five, cryptogenic liver cirrhosis in four, $\alpha$-1-antitrypsin deficiency in two, cystic fibrosis in two, extrahepatic biliary atresia in one and cystic biliary disease in one). The mean age of the patients was 48 years (range 26-69). The Child-Pugh score was obtained by summing up the points for each variable: $(a)$ ascites (none $=1$, slight $=$ 2 , moderate to severe $=3) ;(b)$ encepahalopathy (none $=1$, grade I or II $=2$, grade III or IV = 3); (c) serum bilirubin $(\mathrm{mmol} / \mathrm{l})(0-34=1$, 35-51 = 2, >52 = 3); (d) serum albumin $(\mathrm{g} / \mathrm{l})$ $(>36=1,28-35=2,<28=3) ;(e)$ prothrombin time (seconds) $(<15=1,<20$ but $\geqslant 15=2, \geqslant 20=3)$. The livers were preserved for 13.6 (0.4) hours (mean (SEM); range 9.5-17 hours) in University of Wisconsin solution. During surgery and for the first 24 hours after surgery, lactated Ringer's solution and blood products were administered as needed. A $5 \%$ glucose solution was administered on postoperative days 1 and 2 . Insulin was required in a few patients after day 1 . All patients received standard immunosuppression treatment consisting of low dose predonisolone and cyclosporine or Tacrolimus.

Arterial blood samples were obtained through a catheter inserted for monitoring blood pressure before the operation. The samples were collected in tubes kept on ice and centrifuged at $0^{\circ} \mathrm{C}$, and the serum and plasma were stored at $-70^{\circ} \mathrm{C}$ until assayed. Blood samples were obtained from the 20 healthy controls for $\mathrm{HGF}$ concentration analysis. Serum HGF concentrations were determined using an enzyme linked immunosorbent assay kit (Otsuka Assay, Tokushima, Japan). Glucose concentrations were determined on a neutralised perchloric acid filtrate of plasma using standard enzymatic methods. The plasma levels of immunoreactive insulin were determined by radioimmunoassay using an insulin kit obtained from Sereno Diagnostics (Braintree, Massachusetts, USA). Total ketone bodies were measured by an enzymatic method using highly purified 3-hydroxybutyrate dehy-

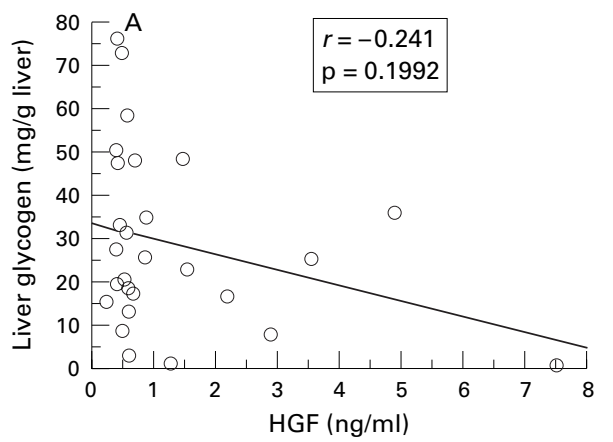

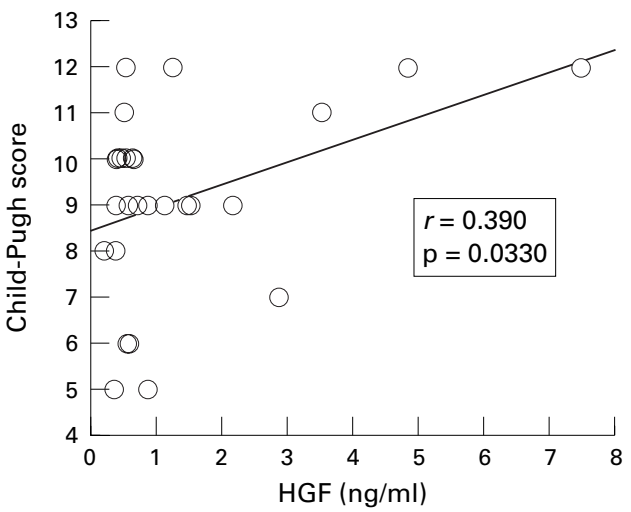

Figure 1 Relation between serum hepatocyte growth factor (HGF) levels and Child-Pugh scores in patients awaiting liver transplantation $(n=30)$.

drogenase, based on the methods of Melanby and Williamson. ${ }^{14}$ Plasma concentrations of total carnitine were determined by a radioenzymatic assay (Kainos, Tokyo, Japan). Plasma amino acid concentrations were determined in plasma deproteinised with $10 \%$ sulphosalicylic acid using high performance liquid chromatography on an amino acid analysing system JLC automatic analyser (Nihon Denshi, Tokyo, Japan). The following were determined: aromatic amino acids (AAAs; tyrosine + phenylalanine), branched chain amino acids (BCAAs; valine + isoleucine + leucine), and the BCAA to AAA molar ratio. Arterial blood samples were also obtained during the anhepatic phase just before graft reperfusion for analysis of plasma amino acid concentrations.

A biopsy specimen was taken from each recipient's rectus muscle at the incision site of the operative wound. A wedge biopsy specimen was taken from the recipient's explanted liver soon after its removal. The wet weights of the biopsy specimens were immediately recorded. The specimens were then immediately frozen in liquid nitrogen and stored at $-70^{\circ} \mathrm{C}$ until assayed. The glycogen contents of these specimens were determined as follows. Briefly, the specimens were homogenised with amyloglucosidase after correction for tissue free glucose, and the increase in NADPH was then measured fluorimetrically.

Informed consent to participate in the study was obtained from each subject. The results are presented as means (SEM). Differences in HGF concentration between patients and con-

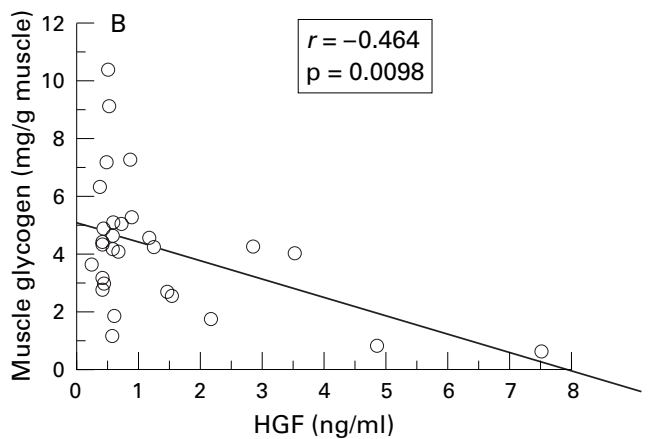

Figure 2 Relation between preoperative serum hepatocyte growth factor (HGF) levels and glycogen content of explanted liver $(A)$ and muscle $(B)$ from liver transplant patients $(n=30)$. 

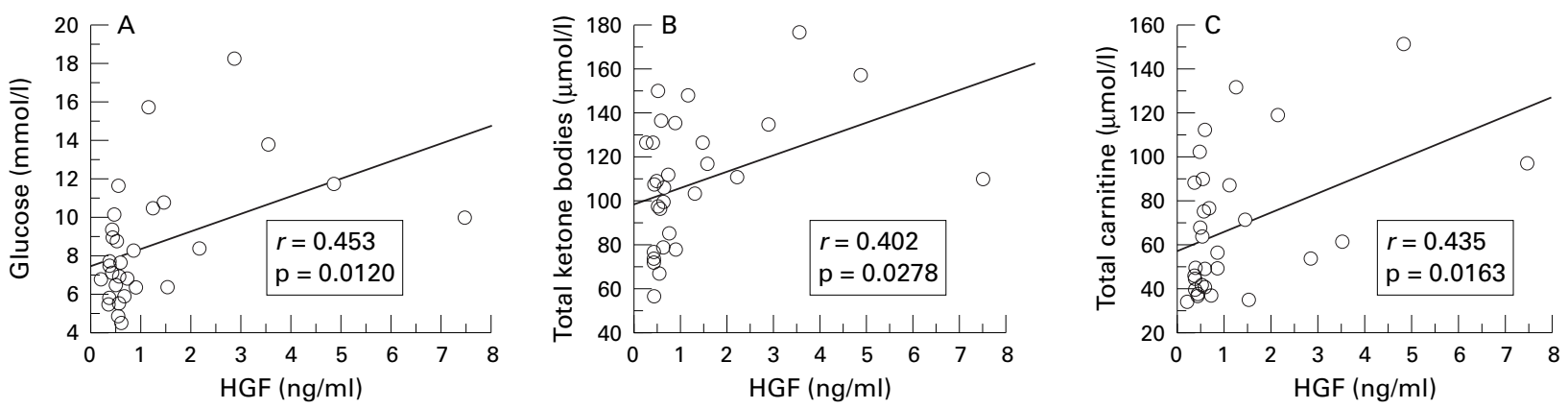

Figure 3 Relation between serum levels of hepatocyte growth factor (HGF) and glucose (A), total ketone bodies (B), and total carnitine (C) in patients awaiting liver transplantation $(n=30)$.
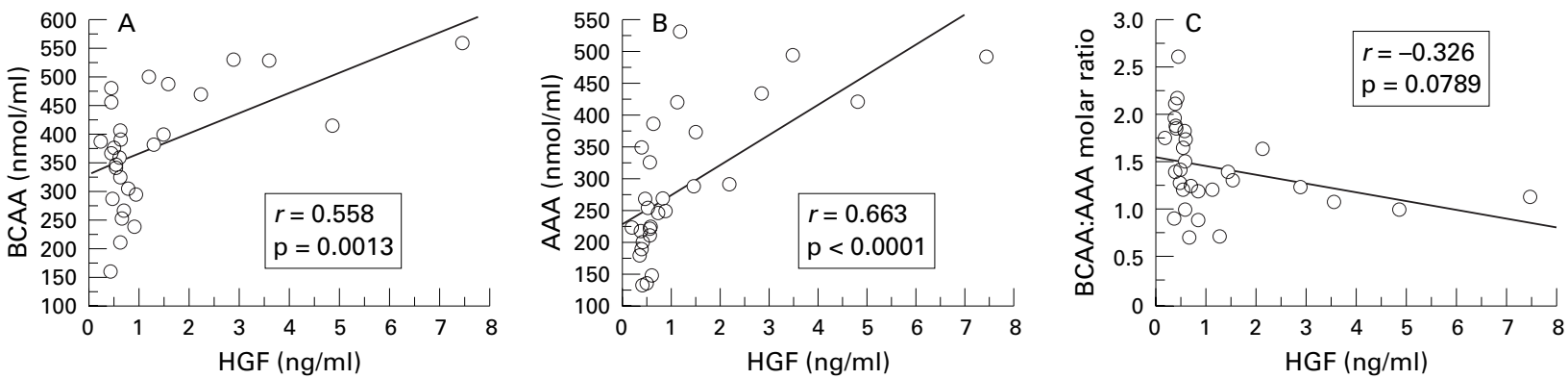

Figure 4 Relation between serum levels of hepatocyte growth factor (HGF) and preoperative plasma levels of branched chain amino acids (BCAA) (A), aromatic amino acids $(A A A)(B)$, and the $B C A A$ to $A A A$ molar ratio $(C)$ in patients awaiting liver transplantation $(n=30)$.

trols were assessed using the Mann-Whitney U test. In a univariate analysis, preoperative levels of $\mathrm{HGF}$ and various variables were analysed. Intragroup differences were compared using the Wilcoxon signed rank test. $\mathrm{p}<0.05$ was considered to be significant.

\section{Results}

The mean serum HGF concentration of the patients was significantly higher than that of the controls $(0.82(0.19) v 0.17(0.01) \mathrm{ng} / \mathrm{ml}$, $\mathrm{p}<0.0001)$. The cut off value was set at 0.5 $\mathrm{ng} / \mathrm{ml}$ based on control values (>mean +4 SD). Serum HGF concentration was elevated in 22 of 30 patients $(73 \%)$. One patient whose preoperative HGF level was the highest of all the patients $(7.49 \mathrm{ng} / \mathrm{ml})$ died 38 days after the transplantation with multiorgan failure resulting from initial poor graft function. Two patients with serum HGF concentrations above $1.0 \mathrm{ng} / \mathrm{ml}$ had a septic complication. The other 27 patients survived without morbidity. Serum HGF concentration correlated with the Child-Pugh score (fig 1).

Figure 2 shows the relation between serum HGF concentration and glycogen content of muscle and explanted liver. The normal range of glycogen content of skeletal muscle is 10-60 $\mathrm{mg} / \mathrm{g}$ muscle. ${ }^{15}{ }^{16}$ Serum HGF concentration correlated with muscle glycogen content, but not with the glycogen content of the explanted liver.

Figure 3 shows the relation between serum $\mathrm{HGF}$ concentration and each of the variables reflecting energy metabolism of the patients. Serum HGF concentration correlated with serum levels of glucose, total ketone bodies, and total carnitine.

Figure 4 shows the relation between serum HGF concentration and preoperative plasma amino acid levels. Serum HGF concentration

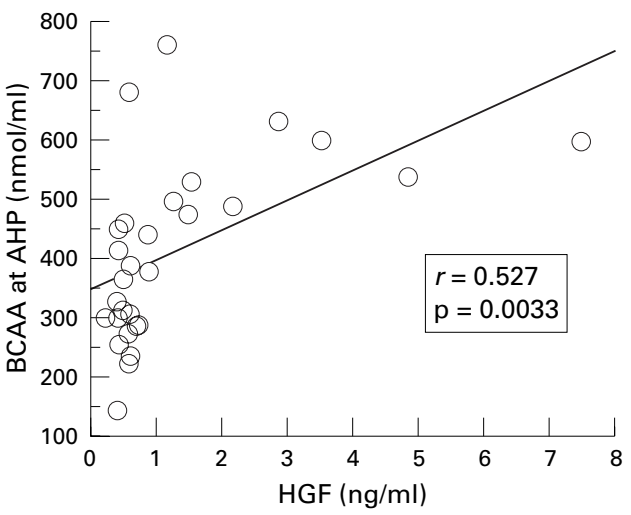

Figure 5 Relation between serum hepatocyte growth factor (HGF) levels and plasma levels of branched chain amino acids (BCAA) at the end of the anhepatic phase (AHP) in liver transplant patients $(n=30)$.

correlated positively with plasma levels of AAAs and BCAAs, and showed a weak negative association with the BCAA to AAA molar ratio.

The plasma BCAA level in patients whose HGF level was above or equal to the cut off value showed a further increase during the anhepatic phase (379.1 (21.0) v 441.0 (32.0) $\mathrm{nmol} / \mathrm{ml}, \mathrm{p}=0.0142$ ), whereas the plasma BCAA level in those whose HGF level was below the cut off value decreased significantly at the end of the anhepatic phase (359.8 (34.8) v 311.4 (37.9) $\mathrm{nmol} / \mathrm{ml}, \mathrm{p}=0.0180)$. The preoperative serum HGF concentration correlated significantly with the plasma BCAA level at the end of the anhepatic phase (fig 5).

\section{Discussion}

Serum HGF concentration is increased in a variety of liver diseases in which there is liver regeneration. Cirrhotic patients with modified 
Child class $\mathrm{C}$ had higher levels of serum HGF than those graded as modified Child class A or B. Serum HGF levels were significantly related to the histological activity index score in the patients with chronic hepatitis. ${ }^{17} 18$ Several studies have also shown the clinical value of measuring serum HGF concentration for predicting outcome in patients with fulminant hepatic failure. ${ }^{1920}$ As an increase in serum HGF concentration in patients with liver disease may be caused by both an increase in $\mathrm{HGF}$ production and a decrease in $\mathrm{HGF}$ clearance by the damaged liver, the increase in HGF activity may well reflect a decrease in functional liver mass. ${ }^{20}$

Carbohydrate metabolism is altered in end stage liver disease. Decreased carbohydrate stores in the liver and muscle are due to accelerated glycogenolysis and impaired glycogenesis as the result of increased insulin insensitivity. ${ }^{2122}$ Recent studies have shown that muscle glycogen content in patients with liver cirrhosis is positively correlated with the resting whole body respiratory quotient, ${ }^{15}{ }^{23}$ suggesting that a depletion of glycogen stores and increased lipid oxidation are the characteristics of tissue catabolism in cirrhotic patients. ${ }^{24}$

In this study, serum HGF concentration showed a negative association with muscle glycogen content. Moreover, it showed a positive correlation with serum levels of glucose, total carnitine, and total ketone bodies. Carnitine plays an essential role in the transport of fatty acids in mitochondria for subsequent oxidation and energy production. It is synthesised mainly in the liver and released into the circulation; changes in plasma carnitine concentration seem to reflect carnitine productivity in the liver. ${ }^{25}{ }^{26}$ It has a stimulating dose-dependent effect on the production of ketone bodies and raises the $\mathrm{NADH}$ to $\mathrm{NAD}^{+}$ratio in liver mitochondria. ${ }^{27}{ }^{28}$ Ketone bodies are generated with acetyl-CoA derived from fatty acids in the liver. The damaged liver then uses fatty acids predominantly to compensate for the persistent inhibition of the tricarboxylic acid cycle. ${ }^{29}{ }^{30}$ As an increase in blood ketone bodies indicates acceleration of fatty acid oxidation, our results may indicate that the serum HGF level reflects extensive preoperative energy catabolism in patients awaiting liver transplantation.

This study also shows that patients with elevated serum HGF concentrations had higher preoperative plasma AAA and BCAA levels, associated with lower BCAA to AAA ratios. Moreover, the plasma BCAA concentration in these patients showed a further increase during the anhepatic phase. BCAAs become an important fuel source in peripheral tissues in cirrhotic patients. Under conditions of metabolic stress, skeletal muscles show an increased demand for BCAAs and use them primarily as a carbon source in oxidative energy production. ${ }^{31}$ In addition, the systemic hyperinsulinaemia observed in advanced chronic liver disease drives plasma BCAAs into the peripheral tissues. Thus the significant decrease in plasma BCAA level in the present patients with high serum HGF can be interpreted as indicating an increase in peripheral utilisation of BCAAs. ${ }^{32-35}$
The increased plasma AAA levels caused by increased degradation of muscle protein and decreased metabolism in the liver result in a depressed BCAA to AAA ratio in these patients. ${ }^{3637}$ The BCAA to AAA ratio is normally 3.0 to 3.5 , but in the setting of liver failure this ratio falls, typically below 2.0. In contrast, increased levels of BCAAs and AAAs were observed in patients with fulminant hepatic failure. ${ }^{38}$ Increased plasma BCAA levels associated with hyperaminoacidaemia in patients with acute liver failure have been reported to indicate a poor prognosis. ${ }^{39}$ As BCAAs leave the hepatic veins after little or no uptake by the liver cells, ${ }^{40}$ and as increased plasma BCAA levels indicate that the peripheral release of BCAAs (principally in muscle tissues) prevails over the increase in the peripheral utilisation of BCAAs, ${ }^{31}$ marked increases in BCAAs and AAAs in the plasma suggest that, at this stage, amino acid metabolism is seriously disturbed not only in the liver but also in other tissues.

In conclusion, the elevated serum concentration of HGF in the liver transplant candidates reflected inhibition of peripheral glucose storage, enhanced lipid oxidation, and increased peripheral release of BCAAs. Serum HGF may be a useful index for evaluating the energy catabolism of patients awaiting liver transplantation.

1 Selberg O, Bottcher J, Tusch G, et al: Identification of highand low-risk patients before liver transplantation: a prospective cohort study of nutritional and metabolic parameters in 150 patients. Hepatology 1997;25:652-7.

2 Hehir DJ, Jenkins RL, Bistrain BR, et al. Nutrition in patients undergoing orthotopic liver transplant. $7 P E N ~ f$ Parenter Enteral Nutr 1985;9:695-700.

3 Delafosse B, Faure JL, Bouffard Y, et al. Liver transplantation: energy expenditure, nitrogen loss, and substrate oxidation rate in the first two postoperative days. Transplant Proc 1989:21:2453-4.

4 Pikul J, Sharpe MD, Lowndes R, et al. Degree of preoperative malnutrition is predictive of postoperative morbidity and mortality in liver transplant recipients. Transplantation 1994;57:469-71.

5 Chin SE, Shephered RW, Thomas BJ, et al. The nature of malnutrition in children with end-stage liver disease awaiting orthotopic liver transplantation. Am $f$ Clin Nutr 1992;56:164-8.

6 Noji S, Tashiro K, Koyama E, et al. Expression of hepatocyte growth factor gene in endothelial and Kupffer cells of damaged rat livers, as revealed by in situ hybridization. Biochem aged rat livers, as revealed by in situ hyt
Biopys Res Commun 1990;173:42-7.

7 Stoker M, Gheradi E, Perryman M, et al. Scatter factor is a fibroblast-derived modulator of epithelial cell mobility. Nature 1987;327:239-42.

8 Cooper CS. The met oncogene: from detection by transfection to transmembrane receptor for hepatocyte growth factor. Oncogene 1992;7:3-7.

9 Kaneko A, Hayashi N, Tanaka Y, et al. Changes in serum human hepatocyte growth factor levels after transcatheter arterial embolization and partial hepatectomy. Am $\mathcal{f}$ Gastroenterol 1992;87:1014-17.

10 Tsubouchi H, Niitani Y, Hirono S, et al. Levels of the human hepatocyte growth factor in serum of patients with various liver diseases determined by an enzyme-linked immunoliver diseases determined by an enzyme
sorbent assay. Hepatology 1991;13:1-5.

11 Fukuura T, Miki C, Inoue T, et al. Serum hepatocyte growth factor as an index of disease status of patients with colorecfactor as an index of disease status of patients

12 Miki C, Iriyama K, Strain A, et al. The clinical significance of serum hepatocyte growth factor in orthotopic liver transplantation. Surgery 1996;119:505-10.

13 Nozu F, Takeyama N, Tanaka T. Changes of hepatic fatty acid metabolism produced by chronic thioacetamide administration in rats. Hepatology 1992;15:1099-106.

14 Melanby J, Williamson DH. Acetoacetate. In Bergmeyer HU, ed. Methods of enzymatic analysis. Orlando, FL: Academic Press, 1974:1840-3.

15 King RF, Macfie J, Hill GL, et al. Effect of intravenous nutrition, with glucose as the only calorie source, on muscle glycogen. 7 PEN $\mathcal{F}$ Parenter Enteral Nutr 1981;5:226-9.

16 Selberg O, Radoch E, Walter GF, et al. Skeletal muscle glycogen content in :epatic failure. Hepatology patient with cirrhosis. Hepatology 1994;20:135-41. 
17 Shiota G, Umeki K, Okano J, et al. Hepatocyte growth factor and acute phase proteins in patients with chronic liver

18 Shioata G, Okano J, Kawasaki H, et al. Serum hepatocyte growth factor levels in liver diseases: clinical implications. Hepatology 1995;21:106-12.

19 Tsubouchi H, Kawakami S, Hirono S, et al. Prediction of outcome in fulminant hepatic failure by serum human hepatocyte growth factor. Lancet 1992;340:307.

20 Tsubouchi H, Hirono S, Gohda E, et al. Clinical significance of human hepatocyte growth factor in blood from patients with fulminant hepatic failure. Hepatology 1989;9:875-81.

21 Muller MJ. Hepatic energy and substrate metabolism: a possible metabolic basis for early nutritional support in cirrhotic patients. Nutrition 1998;14:30-8.

22 Meyer-Alber A, Hartmann H, Stumpel F, et al. Mechanism of insulin resistance in $\mathrm{CCl}_{4}$-induced cirrhosis of rats. Gasof insulin resistance in $\mathrm{CCl}_{4}-\mathrm{in}$.
troenterology $1992 ; 102: 223-9$.

23 Krahenbuhl S, Weber FL, Brass EP. Decreased hepatic glycogen content and accelerated response to starvation in rats
with carbon tetrachloride-induced cirrhosis. Hepatology with carbon tetrach

24 Muller MJ, Dettmer A, Tettenborn M, et al. Metabolic, endocrine, haemodynamic and pulmonary responses to different types of exercise in individuals with normal or reduced liver function. Eur f Appl Physiol 1996;74:246-57.

25 Stanley CA. New genetic defects in mitochondrial fatty acid oxidation and carnitine deficiency. Adv Pediatr 1987;34:5988.

26 Schmidt-Sommerfeld E, Penn D, Kerner J, et al. Quantitation of urinary carnitine esters in a patient with medium-chain acyl-coenzyme dehydrogenase deficiency: effect of metabolic state and L-carnitine therapy. $f$ Pediat 1989;115:577-82.

27 Bohles HJ, Akcetin Z. Ketogenic effects of low and high levels of carnitine during total parenteral nutrition in the rat. Am $\mathcal{f}$ Clin Nutr 1987;46:47-51.

28 O'Connor JE, Costell M, Miguez MP, et al. Effect of L-carnitine on ketone bodies, redox state and free amino acids in the liver of hyperammonemic mice. Biochem Pharmacol 1987;36:3169-73.
29 Takada Y, Ozawa K, Yamaoka Y, et al. Arterial ketone body ratio and glucose administration as an energy substrate in relation to changes in ketone body concentration after living-related liver transplantation in children. Transplantation 1993;55:1314-19.

30 Asonuma K, Takaya S, Selby $\mathrm{R}$, et al. The clinical significance of the arterial ketone body ratio as an early indicator of graft viability in human liver transplantation. Transplantation 1991;51:164-71.

31 Yamaguchi T, Shimahara Y, Takada Y, et al. Plasma amino acid profile in relation to arterial ketone body ratio in surgical patients. Circulation Shock 1993;40:258-63.

32 Francavilla A, Polimeno L, Van Thiel DH, et al. Pancreatic hormones and amino acid levels following liver transplantation. Hepatology 1987;5:918-24.

33 Montanari A, Simoni I, Vallisa D, et al. Free amino acids in plasma and skeletal muscle of patients with liver cirrhosis. Hepatology 1988;8: 1034-9.

34 Shaw S, Lieber CS. Plasma amino acids in the alcoholic: nutritional aspects. Alcohol Clin Exp Res 1983;7:22-7.

35 MacLean DA, Spriet LL, Hultman E, et al. Plasma and muscle amino acid and ammonia responses during prolong

36 Reilly JI, Halow GM, Gerhardt AL, et al. Plasma amino acids in liver transplantation: correlation with clinical outacids in liver transplantation: cor

37 Tessari P, Zanetti M, Barazzoni R, et al. Response of phenylalanine and leucine kinetics to branched chain-amino acids and insulin in patients with cirrhosis. Gastroenterology 1996;111:127-37.

38 Fiaccadori F, Pedretti G, Ferrari C, et al. Insulin and glucagon levels in fulminant hepatic failure in man. Dig Dis $\mathrm{Sci}$ 1991;36:801-8

39 Record CO, Buxton B, Chase RA, et al. Plasma and brain amino acids in fulminant hepatic failure and their relationship to hepatic encephalopathy. Eur f Clin Invest 1976;6: 387-94.

40 Munoz SJ, Jarrell BE, Westerberg S, et al. Serum amino acids following human orthotopic liver transplantation. Transplant Proc 1993;25:1779-82. 